\title{
Homograft Treatment for Infected Frozen Elephant Trunk Prosthesis
}

\author{
Petar Risteski, MD, ${ }^{1}$ Razan Salem, MD, ${ }^{1}$ Thomas Walther, MD, PhD,${ }^{1}$ Johanna Kessel, MD, ${ }^{2}$ \\ Wolf Otto Bechstein, $\mathrm{MD}, \mathrm{PhD}^{3}$ \\ ${ }^{1}$ Department of Thoracic and Cardiovascular Surgery, University Hospital Frankfurt, Frankfurt am Main, Germany; \\ ${ }^{2}$ Department II of Internal Medicine, Infectiology, University Hospital Frankfurt, Frankfurt am Main, Germany; ${ }^{3}$ Department \\ of General and Visceral Surgery, University Hospital Frankfurt, Frankfurt am Main, Germany
}

\section{ABSTRACT}

A 46-year-old male received total arch replacement with frozen elephant trunk for acute non-A/non-B aortic dissection. Two months later, he underwent emergency reoperation for contained rupture of the left common carotid ostium at its insertion on the aortic arch. Three months after the reoperation, he developed tracheoesophageal fistula and infection of the prosthesis in the region of the aortic arch and the proximal descending aorta. Second reoperation was performed with replacement of the aorta with a composite of three aortic homografts, and the fistula was permanently closed with a direct suture and intercostal muscle flap.

\section{INTRODUCTION}

Aortic graft infection is a life-threatening complication that poses a treatment challenge in the absence of international guidelines and scarcity of multicenter studies [Smeds 2016].

Complete removal of the infected aortic graft is the optimal therapeutic approach. Redo surgery to explant the infected graft, especially if a hybrid prosthesis has been used, is associated with increased perioperative risks [Touma 2014].

\section{CASE REPORT}

A 46-year-old male received total arch replacement with frozen elephant trunk (E-vita Open Plus hybrid prosthesis, CryoLife JOTEC GmbH, Hechingen; Germany) for acute non-A/non-B aortic dissection with true lumen collapse.

One week after discharge, he presented with constipation and reported exaggerated Valsalva maneuvers to relieve himself, in addition to fever. Blood cultures revealed Staphylococcus auricularis, Streptococcus mitis, and constellatus. A computed tomography (CT) scan was unremarkable. The bacteremia was successfully treated with i.v. antibiotics.

Received fuly 31, 2020; accepted August 13, 2020.

Petar Risteski, MD, FETCS, Department of Thoracic and Cardiovascular Surgery, University Hospital Frankfurt, Theodor Stern Kai 7, 60590 Frankfurt am Main, Germany; +49-69-6301-5850; fax: +49-69-6301-5849 (e-mail: petarristeski@me.com).
Three weeks after admission, the patient showed severe hemoptysis. An esophagogastroduodenoscopy revealed Mallory-Weiss lesions and the active bleeding was stopped by endoscopic clip application. A CT scan revealed contained rupture of the aortic arch. During emergency reoperation, contained rupture of the left common carotid ostium at its insertion at the aortic arch was confirmed and repaired. In addition, we completely excised the aortic arch graft and replaced it with a new polyester graft. After a course of 6 weeks of antibiotics (Ampicillin, Gentamicin, and Rifampin) and repetitive sterile blood cultures, the patient was discharged in good condition.

Three months later, he suffered fever and fatigue. Streptococcus constellatus was detected in four consecutive blood cultures. Therapy with Ampicillin, Gentamicin, and Rifampin was started again and a fluordesoxyglucose-position emissions tomography (FDG-PET) scan showed abnormal uptake in the aortic graft (Figure 1).

Severe coughing and hemoptysis required bronchoscopy and esophagogastroduodenoscopy that suggested tracheoesophageal fistula, which was later confirmed with dye leakage in the bronchoscopic investigation.

The second redo surgery was carried out five months later through a repeat resternotomy and left anterolateral thoracotomy. Organ protection was provided with moderate systemic hypothermia at $26^{\circ} \mathrm{C}$ using bilateral antegrade brain perfusion. Complete removal of the polyester
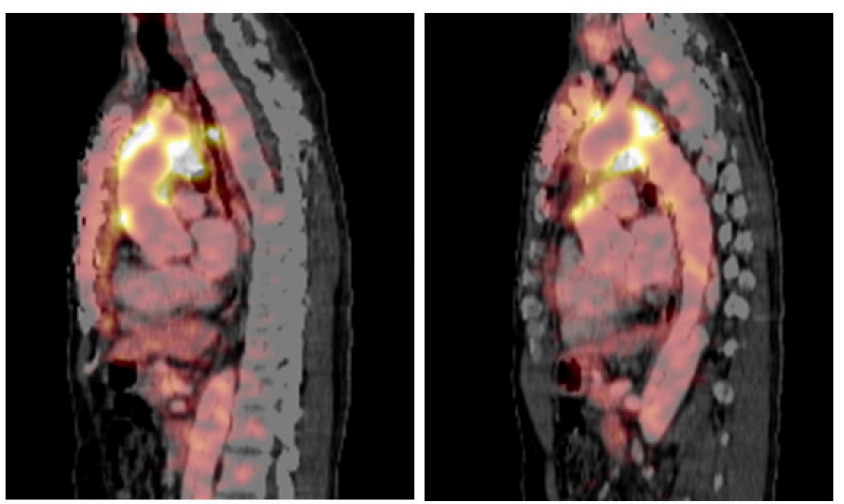

Figure 1. Enhanced glucose uptake on position emissions tomography scan in the region of ascending aorta and proximal aortic arch (left) and distal aortic arch (right). 


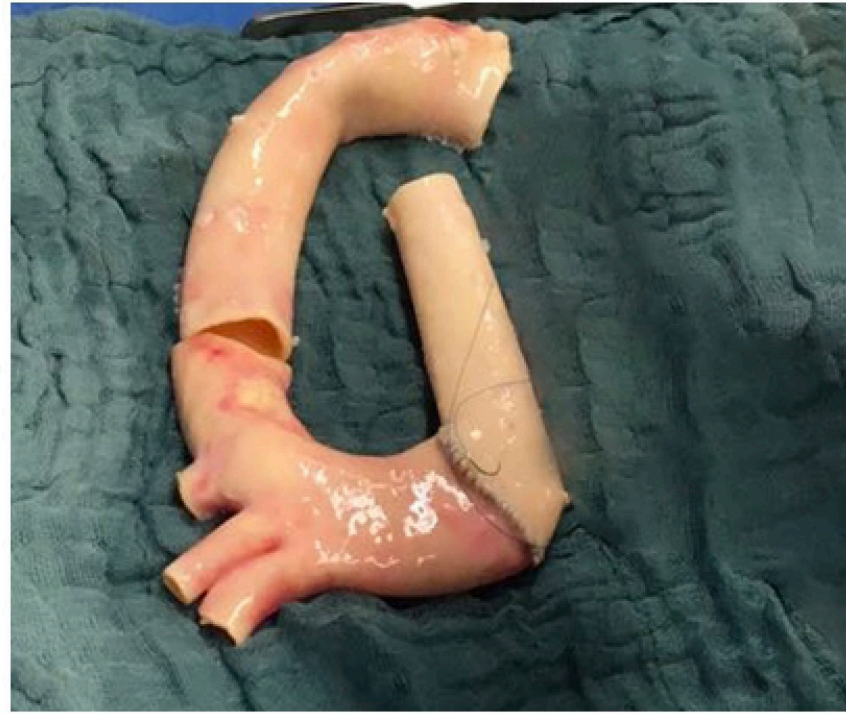

Figure 2. Assembling a thoracic aorta from three homografts on the back table.

prosthesis in the ascending and arch position and resection of the proximal two-thirds of the stent portion of the hybrid prosthesis were performed with debridement and antiseptic irrigation. The distal stent portion of the hybrid prosthesis was left intact, after securing a long safety margin away from the infected site. Once lower body perfusion [Cappabianca 2017] was established, the tracheoesophageal fistula was repaired with isolation and excision, and closure of the esophageal lesion was performed. A generous intercostal muscle flap was wrapped between the esophagus and the trachea to ensure durable closure. The aortic continuity was reconstructed with 3 aortic homografts (Figure 2).

The patient was weaned from the ventilator on the 4th postoperative day. Microbiological examination of the intraoperative material was negative. Histologic report showed signs of unspecific chronic granuloma. At 6-month follow-up, there were no recurrent local or systemic signs of infection, including unremarkable control CT scan (Figure 3).

\section{DISCUSSION}

Graft infection is a disastrous complication after aortic repair with reported incidence of $0.2-5 \%$ and morbidity rates exceeding 35\% [Cappabianca 2017; Heyes 2009; Kirkwood 2010; Smeds 2016; Touma 2014.]

The presence of tracheoesophageal fistula adds to this treatment challenge. Most experts recommend explantation of the infected graft, extensive debridement followed by aortic reconstruction with preferably homografts or xenopericardial tube grafts [Zientara 2016], in addition to longterm antibiotic treatment [Hodgkiss-Harlow 2011]. Interventional treatment of tracheoesophageal fistula by double lumen stenting with self-expanding covered stents may be

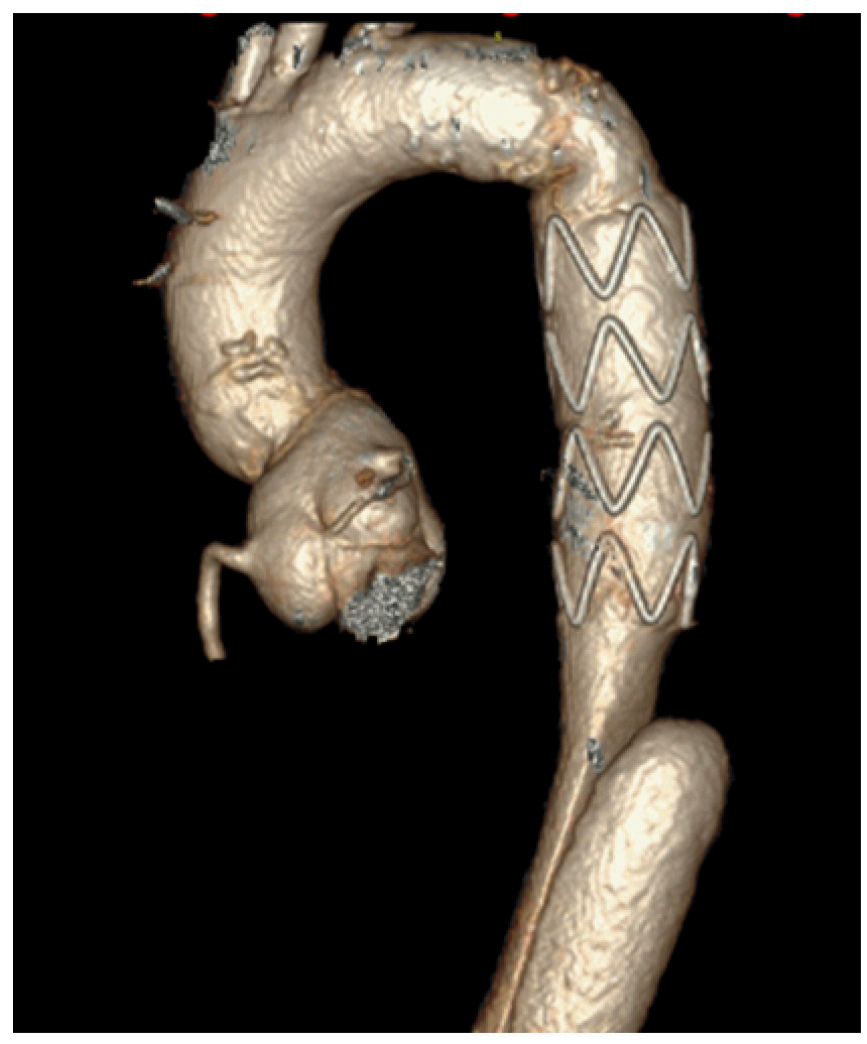

Figure 3. Final result after operative treatment of infected frozen elephant trunk prosthesis.

used as temporary but not definitive treatment. If the lesion in the esophagus can be closed without tension after debridement, this should be attempted. The defect in the trachea will close spontaneously after safe closure of the esophageal defect and interposition of vital tissue, as happened eventually in the case described.

Safe re-entry into the chest, good organ protection and perfusion, durable treatment of the fistula, and careful selection of replacement material are the backbone of successful treatment. We left the distal part of the stent in the descending aorta to keep the true lumen open and have an ideal landing zone for future stent implantations.

The duration of antibiotics is another debated issue. Our patient was closely co-advised by the infectious disease consultancy service since his first fever episode. Although agreement exists on a combination of two or more bactericidal drugs that penetrate bacteria biofilms [Hodgkiss-Harlow 2011], opinions vary between 3-6 months, or longer administration of antibiotics [Heyes 2009; Hodgkiss-Harlow 2011; Kirkwood 2010; Smeds 2016; Touma 2014]. We chose 6-months enteral and parenteral double bactericidal treatment followed by a search for subclinical, radiologic, and clinical evidence of persistent infection.

By means of a structured interdisciplinary team approach, as shown with our patient, even extreme, complex situations can be successfully treated. 


\section{REFERENCES}

Cappabianca G, Roscitano C, Bichi S, et al. 2017. Whole body perfusion in patients undergoing frozen elephant trunk for type A acute aortic dissections. Perfusion 32:164-7.

Heyes KS, Modi P, Morasch MD, et al. 2009. Secondary infection of thoracic and abdominal aortic endografts. J Vasc Interv Radiol 20:173-9.

Hodgkiss-Harlow KD, Bandyk DF. 2011. Antibiotic therapy of aortic graft infection: treatment and prevention recommendations. Semin Vasc Surg 24:191-8.
Kirkwood ML, Pochettino A, Fairman RM, et al. 2010. Thoracic aortic endograft explant: a single-center experience. Vasc Endovascular Surg 44:440-5.

Smeds MR, Duncan AA, Harlander-Locke MP, et al. 2016. Treatment and outcomes of aortic endograft infection. J Vasc Surg 63:332-40.

Touma J, Cochennec F, Parisot J, et al. 2014. In situ reconstruction in native and prosthetic aortic infection using cryopreserved arterial allografts. Eur J Vasc Endovasc Surg 48:292-9.

Zientara A, Schwegler I, Dzemali O, et al. 2016. Xenopericardial selfmade tube grafts in infectious vascular reconstruction: Preliminary results of an easy and ready to use surgical approach. Vascular 24:621-7. 